\title{
Primary Open Angle Glaucoma in the Young Age
}

\author{
Ahmed Mostafa Abdelrahman ${ }^{1 *}$ and Rana H Amin ${ }^{2}$ \\ ${ }^{1}$ Professor of Ophthalmology and Glaucoma Consultant, Kasr Aini School of Medicine, \\ Cairo University, Egypt \\ ${ }^{2} \mathrm{MSc}$, Cairo University, Egypt \\ *Corresponding Author: Ahmed Mostafa Abdelrahman, Professor of \\ Ophthalmology and Glaucoma Consultant, Kasr Aini School of Medicine, Cairo \\ University, Egypt.
}

Received: March 13, 2020

Published: April 09, 2020

(C) All rights are reserved by Ahmed Mostafa

Abdelrahman and Rana $\mathrm{H}$ Amin.
Primary open angle glaucoma in the young age is known as The Juvenile open angle glaucoma (JOAG) which is a form of primary open angle glaucoma (POAG) that presents with elevated IOP intraocular pressure between the ages of 4 and 35 years (up to 40 years of age in other reports). Most cases are inherited as an autosomal dominant trait, and therefore, most families may be aware of their risk of developing this condition. In spite of IOP elevation, it doesn't usually cause corneal enlargement or Haab's Striae; rather, progressive myopia may continue [1]. The mean age at diagnosis was $26 \pm 9.8$ years and the estimated prevalence of JOAG ranges from 0.38 to 2 in 100,000 in individuals between 4 and 20 years of age [2].

The elevated IOP in JOAG is attributed to an abnormal trabecular meshwork causing reduced aqueous outflow. Thick compact tissue and extracellular deposits in the trabecular meshwork were detected in individuals with JOAG [3].

JOAG is inherited in an autosomal dominant pattern with high penetrance, of particular importance is Myocilin (MYOC) gene mutations that exceeded 70 and were linked to disease development. Pedigrees with JOAG have shown a locus on chromosome 1q21-31 called GLC1A, that was refined to 1q23-25. Myocilin gene was isolated from the GLC1A locus. Myocilin gene mutations were identified in up to $36 \%$ of individuals with JOAG. Myocilin mutations are also present in adult onset POAG with a prevalence of $4 \%$. Myocilin gene mutations carriers have a 60 - 100\% risk of developing JOAG. However, having the gene mutations does not necessarily mean that the patient has or will develop the disease. Another gene is CYP1B1 (cytochrome P450, family 1, subfamily B, polypeptide 1) that predisposes to JOAG. CYP1B1 was found also found in congenital glaucoma [4-9].

Regarding the risk factors, JOAG patients had a male preponderance, myopic refraction and severe elevation of IOP [10-13]. Afri- can ancestry population has a higher prevalence of JOAG. Prominent iris processes were observed in both affected and unaffected individuals with JOAG. Mutations of Myocilin are another risk factor [14]. Bilateral JOAG patients are older, usually with a positive family history compared to unilateral JOAG patients [15].

JOAG is usually asymptomatic, like POAG, at the time of diagnosis. However, symptoms may include blurred vision, ocular pain, or decreased visual acuity at later stages. Inquiry about the family history of glaucoma is important as well as other history that may indicate a secondary glaucoma [15].

Physical examination and findings of JOAG are similar to POAG. Exam should include measurement of IOP and central corneal thickness as well as the assessment of the anterior chamber angle, optic disc, and visual fields. IOP measurements are done using Goldmann Applanation Tonometry or handheld devices such as the Tono-pen or I- care may be used. Visual field testing using the automated static threshold Perimetry is performed whenever possible. For younger children, Goldmann visual fields may be used. Despite the lack of normative data in young patients, Optical Coherence Tomography (OCT) might be helpful for monitoring the progression. No specific laboratory tests are required [14].

Typically, these patients present optic disc cupping with thinning of the neuroretinal rim and an extremely high IOP, sometimes greater than 40 - $50 \mathrm{mmHg}$. Gonioscopy usually reveals open anterior chamber angles. Other gonioscopic findings includes high iris insertion or prominent iris processes. No other ocular anomalies or systemic disease are detected. As the disease presents after the age of 4 years, there are no signs typically seen in Primary Congenital Glaucoma (PCG) such as corneal edema and enlargement or Haab's striae [14-16].

Evaluating the radial peripapillary capillary plexus using Optical Coherence Tomography Angiography (OCT-A) revealed a sig- 
nificant reduction in the capillary density in patients with JOAG. (Personal, unpublished data).

JOAG should be differentiated from late congenital glaucoma and secondary glaucomas including steroid-induced, traumatic and inflammatory glaucoma. JOAG can be distinguished from late congenital glaucoma by the absence of the classic triad of buphthalmos, tearing, and photophobia. Additionally, cases with JOAG neither show megalocornea nor Descemet's breaks (Haab's striae) that can be seen in primary congenital glaucoma [14,17].

Primary glaucoma at this young age can be further classified according to the age of onset: 1- Early juvenile/Late-onset childhood open angle glaucoma. From 2nd year of life till puberty. The condition resembles primary congenital glaucoma with neither ocular enlargement nor congenital ocular anomalies or syndromes. 2- Primary juvenile glaucoma: usually after puberty [18]. However, there is some overlap when it comes to very young patients 2 - 4 years of age at the time of diagnosis.

In an attempt to re-define POAG, Pasqauel has identified the African-derived type of OAG. The disease is characterized by early onset (demonstrated examples of 32, 34 and 12 year old patients at the time of presentation), $80 \%$ reduced risk of optic disc hemorrhages on examining the optic nerve head, and the familial incidence [19]. The findings are supported by the results of another study which screened an Afro-Carib-bean population living just south of Miami. Their data revealed that almost 21 percent of people between the ages of 20 and 40 had either an IOP $\geq 24$ or a cupto-disc ratio $\geq 0.7$, in at least one eye. This data demonstrated that younger people of African descent can indeed develop glaucoma [20]. Is that African-derived type of OAG is the same as the classic JOAG? A point that needs further investigations.

Generally, the treatment aims at reducing the IOP starting with medical therapy. However, surgery is often required.

Medical therapy includes carbonic anhydrase inhibitors, prostaglandin analogues, beta blockers, and adrenergic agonist. Alphaagonists should be used with caution in small children due to adverse side effects such as central nervous system depression; for this reason, apraclonidine may be preferable to brimonidine in young children with JOAG [14]. Despite the relative safety of apraclonidine over brimonidine, adrenergic agonists are better avoided in children younger than six years and weighting less than $20 \mathrm{Kgs}$ [16].

Surgical intervention is often required and should be carried out by experienced surgeons. Various procedures have been used with varying success rates. Procedures include trabeculectomy, non-penetrating glaucoma surgery, trabectome, glaucoma daring implants, cycloablation and angle surgery including 360 microcatheter trabeculotomy. Also, different combination of techniques have been used [16,21-23].

The choice of the procedure is largely dependent upon the condition of the eye, previous interventions and conjunctival scarring. In addition to that, the surgeon's experience plays a great role in the decision-making process and surgical outcomes. The earlier the diagnosis and the treatment of JOAG, the better the prognosis.

Controversy exists regarding the priority of trabeculectomy as a surgical option for JOAG. Some reports consider trabeculectomy as the mainstay for surgical therapy. Tasi and colleagues have an intermediate-term cumulative success probability of $73 \%$ for the mitomycin-c (MMC) treated group and 68\% for the control group [24]. Pathania and colleagues reported a complete success of $80 \%$ and a qualified success rate of $96 \%$ at five years for trabeculectomy without MMC [25]. On the hand, given the younger age range of JOAG patients and their life expectancy, it is worth considering an alternative to trabeculectomy with antifibrotics, which carries a risk of up to 1.2 percent per year of bleb-associated endophthalmitis [26].

In conclusion, it is important for clinicians to be familiar with JOAG, a glaucomatous optic neuropathy that is usually bilateral, potentially blinding and asymptomatic in most of cases and presents before 35 years of age. It is recommended to check IOP as early as possible in children, especially those with a family history of glaucoma. The IOP is usually very high and the discs are severely damaged at the time of diagnosis. The typical features of PCG as corneal enlargement and Haab's striae are absent. Transmission is autosomal dominant with high penetrance. Surgical intervention is often required. The disease has good prognosis if diagnosed and treated early.

JOAG opens the gates for some questions. What is the role of ethnicity in the disease presentation and prognosis? Is the Africanderived type of OAG the same as the JOAG? Is there a genetic link between POAG and JOAG? What is the best initial surgical option? And how to manage recurrences?

\section{Bibliography}

1. Glaucoma. "Basic and clinical science course 2015-2016.

2. Aponte EP., et al. "Incidence and clinical characteristics of childhood glaucoma: a population-based study". Archives of ophthalmology 128 (2010): 478-482. 
3. Tawara A and Inomata H. "Developmental immaturity of the trabecular meshwork in juvenile glaucoma”. American Journal of Ophthalmology 98.1 (1984): 82-97.

4. Khan AO. "Genetics of primary glaucoma". Current Opinion in Ophthalmology 22.5 (2011): 347-355.

5. Aldred MA., et al. "Low prevalence of MYOC mutations in UK primary open-angle glaucoma patients limits the utility of genetic testing". Human Genetics 115 (2004): 428-431.

6. Fingert JH., et al. "Analysis of myocilin mutations in 1703 glaucoma patients from five different populations". Human Molecular Genetics 8 (1999): 899-905.

7. Sripriya S., et al. "Low frequency of myocilin mutations in Indian primary open-angle glaucoma patients". Clinical Genetics 65 (2004): 333-337.

8. Shimizu S., et al. "Age-dependent prevalence of mutations at the GLC1A locus in primary open-angle glaucoma". American Journal of Ophthalmology 130 (2000): 165-177.

9. Yen YC., et al. "Identification of mutations in the myocilin (MYOC) gene in Taiwanese patients with juvenile-onset openangle glaucoma". Molecular Vision 13 (2007): 1627-1634.

10. Lotufo D., et al. "Juvenile glaucoma, race, and refraction". Journal of the American Medical Association 261 (1989): 249-252.

11. Goldwyn R., et al. "Primary open-angle glaucoma in adolescents and young adults". Archives of ophthalmology 84 (1970): 579-582.

12. Perkins ES. "Glaucoma in the younger age groups". Archives of ophthalmology 64 (1960): 882-891.

13. Gupta V., et al. "Long-term structural and functional outcomes of therapy in juvenile-onset primary open-angle glaucoma: a five-year follow-up". Ophthalmologica 228 (2012): 19-25.

14. Wong CY., et al. "Juvenile Open Angle Glaucoma. Eye Wiki". American Academy of Ophthalmology (2019).

15. Kwun Y., et al. "Clinical Characteristics of Juvenile-onset Open Angle Glaucoma". Korean Journal of Ophthalmology 30.2 (2016): 127-133.

16. "Primary Congenital glaucoma and Juvenile open-angle glaucoma". In: Weinreb RN, Grajewski A, Papadopoulos M, Grigg J, Freedman S. In Childhood Glaucoma consensus: Krugler publications (2013).
17. "Diagnosing and Managing Juvenile Open-Angle Glaucoma". Written By: Chak G, Mosaed S, Minckler DS, Edited by Ingrid U. Scott IU, Fekrat S. EyeNet Magazine. aao.org. (2014).

18. Terminology and guidelines for Glaucoma. European Glaucoma Society (EOS) $4^{\text {th }}$ Edition (2014).

19. Louis R Pasquale. "Redefining Primary Open-angle Glaucoma”. Review of Ophthalmology (2017).

20. Bokman CL., et al. "Glaucoma screening in the Haitian AfroCaribbean population of South Florida". PLoS One 30.9 (2014): e115942.

21. Stangos AN., et al. "Primary viscocanalostomy for juvenile open-angle glaucoma". American Journal of Ophthalmology 140.3 (2005): 490-496.

22. Abdelrahman AM., et al. "Sutureless Deep Sclerectomy. A preliminary Report". Journal of Glaucoma 26.11 (2017): 255-256.

23. Damji KF., et al. "Efficacy and safety of ab interno trabeculectomy with Trabectome in JOAG". Poster 21. Presented at the $24^{\text {th }}$ annual meeting of the American Glaucoma Society (2013).

24. Tasi JC., et al. "Trabeculectomy with mitomycin $\mathrm{C}$ versus trabeculectomy alone for juvenile primary open-angle glaucoma". Ophthalmologica 217.1 (2003): 24-30.

25. Pathania D., et al. "Outcomes of trabeculectomy in juvenile open- angle glaucoma". Indian Journal of Ophthalmology 62.2 (2014): 224-228.

26. Ang GS., et al. "Postoperative infection in penetrating versus non-penetrating glaucoma surgery". British Journal of Ophthalmology 94.12 (2010): 1571-1576.

\section{Assets from publication with us}

- Prompt Acknowledgement after receiving the article

- Thorough Double blinded peer review

- Rapid Publication

- Issue of Publication Certificate

- High visibility of your Published work

Website: www.actascientific.com/

Submit Article: www.actascientific.com/submission.php Email us: editor@actascientific.com

Contact us: +919182824667 